\title{
Two-Stage Complete Deroofing Fistulotomy Approach for Horseshoe Fistula: Successful Surgery Leaving Continence Intact
}

\author{
Asami Usui, Gentaro Ishiyama, Akihiko Nishio, Maiko Kawamura, Yukiko Kono, Yuji Ishiyama \\ Department of Coloproctology, Sapporo Ishiyama Hospital, Sapporo, Japan
}

Purpose: Surgery of the horseshoe fistula is challenging due to its complex configuration and sphincter muscle involvement. Complete deroofing fistulotomy for horseshoe fistula is highly curative with the eradication of all fistulous lesions but has been discredited for its high incontinence rate. It was replaced with the more conservative Hanley's procedure leaving the lateral tracts intact, despite its issue of recurrence. Our study aimed to report the outcomes of a procedure dividing complete deroofing fistulotomy for horseshoe fistula into 2 stages to avoid impairment of sphincter function. Methods: We retrospectively reviewed 139 patients who underwent surgery for horseshoe fistula using the 2-stage complete deroofing fistulotomy method between 2014 and 2017. The first surgery deroofed the lateral tracts with an arch-like incision severing the anococcygeal ligament. The primary lesion was also drained and curetted. A seton was placed in the primary tract which was laid open in the second surgery after the lateral wound had partially healed.

Results: Recurrence was observed in 12 patients. All were superficial recurrences except for 1 , in which recurrence was confirmed in the primary lesion. Those with blind intersphincteric upward extensions had a significantly higher recurrence rate. Furthermore, patients who resided far from the hospital and could not make visits for frequent wound inspections also had a significantly higher recurrence rate. No patient had any continence issues at the end of the follow-up period. Conclusion: Managing horseshoe fistula with the 2-stage deroofing fistulotomy approach allows for eradication of the fistula tract without compromising anal sphincter function.

\section{Keywords: Horseshoe fistula; Deroofing fistulotomy; Anal fistula}

\section{INTRODUCTION}

Horseshoe fistulas are deep posterior anal fistulas which extend into the ischiorectal space with either unilateral or bilateral extensions in the shape of a horseshoe [1-3]. Surgical management involves eradicating the fistula to prevent recurrence as well as preserving the anal sphincter function, both a challenge in horseshoe

Received: Feb 12, 2020 - Revised: Jun 5, 2020 - Accepted: Jun 8, 2020 Correspondence to: Asami Usui, M.D.

Department of Coloproctology, Sapporo Ishiyama Hospital, 10-4-1 Minami 15-jo Nishi, Chuo-ku, Sapporo 064-0915, Japan

Tel: +81-11-551-2241, Fax: +81-11-551-2727

E-mail: samasamisami@gmail.com

ORCID: https://orcid.org/0000-0003-2217-9534

(C) 2021 The Korean Society of Coloproctology

This is an open-access article distributed under the terms of the Creative Commons Attribution NonCommercial License (https://creativecommons.org/licenses/by-nc/4.0) which permits unrestricted noncommercial use, distribution, and reproduction in any medium, provided the original work is properly cited. fistulas due to its complex spread [4]. Despite its implication on continence, 1-stage deroofing fistulotomy of the entire fistula tract had been widely practiced for a long time when curability was the priority [5], until Hanley [3] introduced a conservative procedure in 1965 where fistulotomy was done only on the primary tract and the lateral tracts are left to heal on its own. These days, a modification of Hanley's procedure in which a delayed fistulotomy on the primary tract is performed with a cutting seton $[4,6]$ seems to be the procedure of choice for horseshoe fistulas, but the problem of recurrence has still remained an issue $[7,8]$. Lateral extensions which are not deroofed have been reported to recur [2]. Recurrence may also be due to difficulty in creating an adequate drainage route for the primary lesion, situated far from the skin surface, with a narrow posterior midline incision.

To eliminate these problems, we modified the highly curative complete deroofing fistulotomy procedure and divided the surgery into 2 stages, so continence is left intact. The first stage elimi- 
nates the infection in both the primary lesion and the extended fistula arms with a wide incision while a seton is placed in the primary fistula around the sphincter complex. The second stage, performed after the supporting tissue surrounding the anus is healed, lays open the primary fistula, dividing the sphincter muscles. The outcomes of this surgical procedure are presented.

\section{METHODS}

We retrospectively analyzed 139 consecutive patients who underwent the 2-staged deroofing fistulotomy for horseshoe fistula between January 2014 and December 2017 at a single institution specializing in proctology. All fistulas indicated for this procedure were transsphincteric fistulas of primary lesion origin extending into the ischiorectal space with either unilateral or bilateral horseshoe arms. None of the patients diagnosed as horseshoe fistula during this study period received other surgical treatments including the original or modified Hanley procedure. Patients with Crohn disease or malignancy in the anus were excluded from the analysis. This study was approved by the local Institutional Review Board which is headed by Sapporo Dohto Hospital, and written informed consent was waived because of the retrospective design. Written consent for the use of clinical images was obtained from all patients. Preoperative diagnosis of horseshoe fistula was made with digital examination and supported with preoperative nonenhanced computed tomography scans and transanal endosonography. The final diagnosis was confirmed with operative findings. Relevant data such as the extent of the pathology were derived from surgical exploration, including palpation and probing.

\section{Surgical procedure}

All abscesses were drained either spontaneously or surgically prior to definitive surgery, and a drainage procedure was carried out if a patient presented with an undrained sepsis. When the abscess was extensive, definitive surgery was performed 3 to 4 weeks after drainage, allowing time for the fistula to form. All patients were given stool softeners the day before definitive surgery as preoperative bowel preparation. Surgery was performed under caudal anesthesia in the jackknife position. A long, arch-like incision was placed over the lateral tracts of the horseshoe, connecting the cutaneous drainage sites when present, so that the whole secondary tract could be deroofed. If the sepsis has been drained internally with no skin markings, the lateral fistula extensions were palpable from inside the anal canal and easily approached cutaneously. All extensions from the lateral tract pursued by probing were curetted and drained. Trimming of the fistula walls to excise fibrosis resulting from inflammation over a prolonged period was done as much as possible without damaging the sphincter muscles. Upon approaching the posterior portion of the lateral tracts over the midline, the anococcygeal ligament appears. In order to obtain a better view of the anatomy behind this, the muscle structure was severed using electrocautery. By deepening this posterior dissection, the primary lesion, which contains granulomatous tissue, was opened up to be curetted. When a blind upward extension from the ischiorectal sepsis running through the levator ani muscle into the supralevator space was present, the opening in the muscle was dilated to facilitate drainage. When a blind intersphincteric upward extension was present, the opening was also dilated, taking care not to damage the rectum wall. After all the lateral tracts and the primary lesion were deroofed, curetted, and drained, a seton using a Penrose drain was inserted in the posterior midline, around the sphincter complex through the primary tract to the internal opening. The seton was not intended as a cutting seton, but as a drainage route to avoid premature closure of the wound before the deep origin was healed. The wound was left open and packed with gauze dressing so that the wound could heal from the bottom up with no dead space left.

Patients were scheduled for the second surgery with a minimum of 5 days from the first surgery, when the tissue surrounding the anus had solidified. Patients were given the option to receive the second surgery in the same admission as the first, or at a later date in another admission more convenient for them. The second surgery was performed with the same preoperative preparations, under the same anesthesia and position. The wound was checked for proper healing as well as new fistulous pockets requiring further drainage. Fistulous pockets usually appeared when drainage was inadequate in the first surgery or premature closure occurred over scarring tissue. If the wound was assessed as not healing properly and required additional drainage of the lesions, the sphincter complex was left intact to be laid open in the next surgery. Otherwise, the seton was removed and the sphincter muscles were divided (Fig. 1). All surgeries were performed by each one of the 4 surgeons with more than 10 years of experience in this anorectal pathology.

The patients were allowed meals on the day of the surgery and kept on bed rest until the next morning. Postoperatively, all patients were prescribed a 3-day course of antibiotics and stool softeners when necessary after each surgery. Analgesics were given regularly for the first 5 days and then as needed afterward. Wound dressings were changed at least twice a day and gauze was packed into the wound to avoid premature closure.

\section{Follow-up}

The first follow-up was scheduled 1 or 2 weeks after surgery, followed by routine visits every 2 to 4 weeks, depending on how far they lived from the outpatient clinic. All patients were questioned for symptoms of anal incontinence at each of the visits and digital anal examination was performed to assess anal tonus. Recurrence was defined as the presence of an abscess occurring after wound closure. Success was defined as the absence of recurrence and the complete healing of the wound.

\section{Statistical analysis}

Continuous data were presented as mean and standard deviation, 

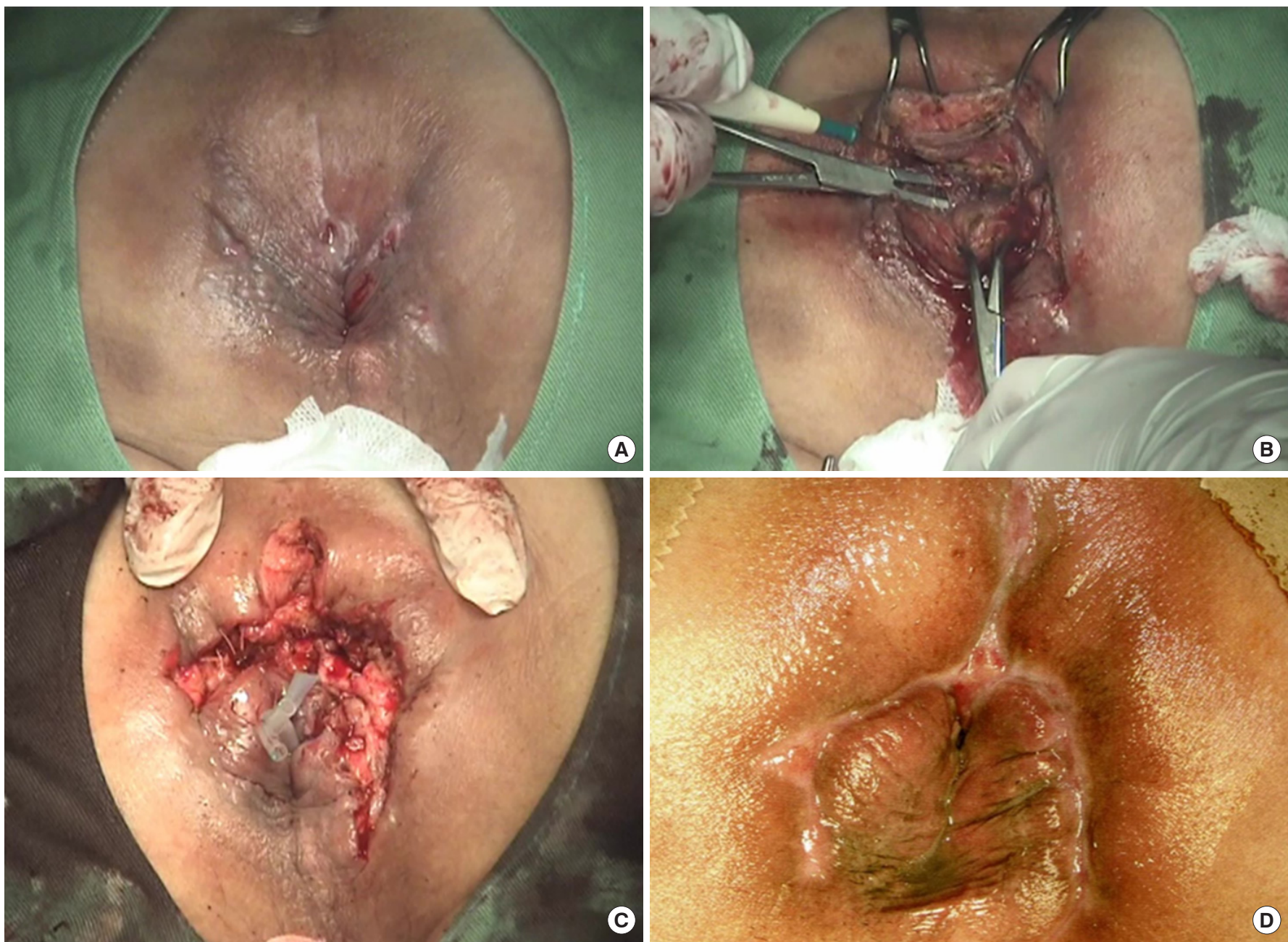

Fig. 1. Two-stage deroofing fistulotomy in a bilateral horseshoe fistula limited to the ischiorectal space. (A) The patient presented with secondary openings on the surface along with the lateral extensions. (B) In the first surgery, the superficial external sphincter muscle is severed and the fistula tracts behind it were deroofed and curetted. (C) After deroofing of the entire lateral extension and drainage of the primary lesion, a seton is placed around the sphincter complex, which is laid open in the second surgery. (D) Five weeks after the second surgery, the wound is healed and deformity to the anus is minimal.

or median and range. Comparisons were made using the chisquared test for categorical variables and the Student t-test for continuous data. P-values less than 0.05 were considered significant.

\section{RESULTS}

There were 122 men and 17 women with a mean age of $47.2 \pm$ 15.4 years. The median duration of disease until surgery was 2.8 months (range, 0.2 to 480 months), and 29 patients (20.9\%) had a duration of more than 1 year. Twenty-one patients (15.1\%) had undergone previous surgery for anal fistula elsewhere, in which details of the operative procedure were unclear. Operative findings (Fig. 2) showed all patients to have a transsphincteric horse- shoe fistula originating from a posterior primary lesion. The fistulous lesion was limited to the ischiorectal space in 111 cases, with the remaining 28 possessing supralevator extensions (14 patients, $10.1 \%$ ), high blind intersphincteric extensions (15 patients, $10.8 \%$ ), or both (1 patient, $0.7 \%$ ). The horseshoe arms extended bilaterally in 87 patients (62.6\%) with the remainder 52 limited to one side. The number of surgeries until the final sphincter division was 2 in 105 patients (75.5\%), 3 in 29 patients (20.9\%), and 4 in 5 patients (3.6\%). One patient with short bilateral extensions had the deroofing fistulotomy done in a single procedure, due to the patient's strong request. The patient was lost to follow-up and the effect on continence of this single procedure could not be confirmed.

The median length of hospital stay for patients whose treatment 


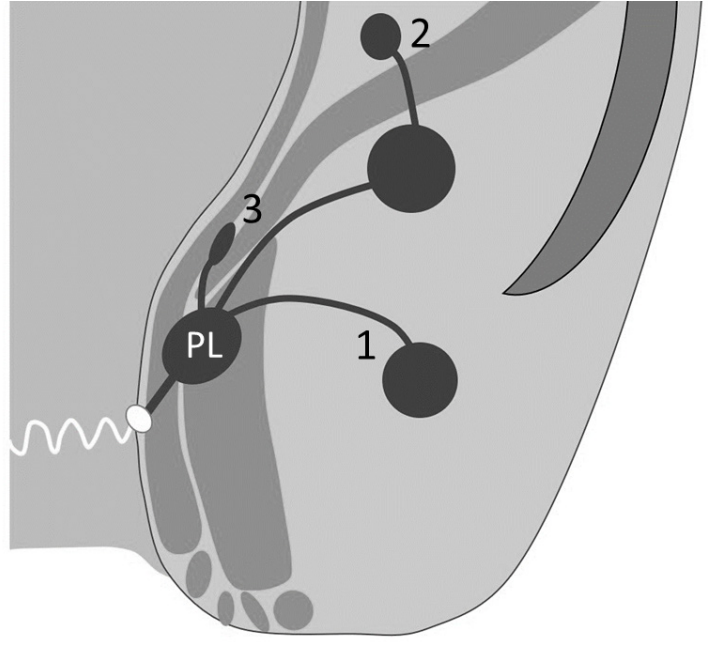

Fig. 2. Schematic diagram of operative findings. All patients were found to have a transsphincteric horseshoe fistula originating from a posterior primary lesion (PL). 1, Limited to the ischiorectal space (111 cases, $79.9 \%)$; 2 , with supralevator extensions (14 patients, $10.1 \%$ ); 3 , with high blind intersphincteric extensions (15 patients, 10.8\%).

consisted of 2 procedures done in one admission was 17 days (range, 13 to 32 days), with a median of 9 days (range, 5 to 14 days) between the first and second surgery. For those who had the surgeries done in separate admissions, the median interval between the procedures was 9.1 weeks (range, 3 to 31.6 weeks). The median time for the wound to heal completely after the final surgery in single admission patients was 13.4 weeks (range, 3.9 to 37.1 weeks), with 4 lost to follow-up prior to confirmation of complete wound healing.

The median follow-up period was 25.1 weeks after the final surgery, ranging from 0 to 252 weeks, with only those seeking care for recurrence or other anal disorders to be long in follow-up. Including the 4 patients who were lost to follow-up after their single admission, there were 7 patients in which complete wound healing could not be confirmed due to loss to follow-up. At the end of the follow-up period, none of the remaining 132 patients had any symptoms of incontinence. During the wound healing process, 3 patients had a Wexner incontinence score of 2 , with symptoms of occasional incontinence of either liquid stool or gas, but all symptoms resolved by 6,8 , and 14 weeks after the final surgery.

The presence or absence of recurrence was documented in the 132 patients who were followed up until complete wound closure and observed in 12 patients (9.1\%). All were superficial recurrences except for 1 , in which recurrence was confirmed as an abscess occurring in the primary lesion. The median time of recurrence was 18.2 weeks (range, 5 to 171 weeks) after the final surgery. All patients received additional surgery to remove the recurring pathology and were healed with no further recurrence. The risk factors for recurrence are shown in Table 1. Analysis showed 3 statistically significant risk factors for recurrence: prolonged du-
Table 1. Risk factors for recurrence

\begin{tabular}{lccr}
\hline \multirow{2}{*}{ Variable } & \multicolumn{2}{c}{ Recurrence } & \multirow{2}{*}{ P-value } \\
\cline { 2 - 3 } & No & Yes & \\
\hline Patient & $120(90.9)$ & $12(9.1)$ & \\
Age (yr) & $48.3 \pm 15.5$ & $41.0 \pm 10.4$ & 0.110 \\
Sex, male:female & $105: 15$ & $11: 1$ & 0.670 \\
Prolonged symptom duration (mo) & $22(18.3)$ & $6(50.0)$ & 0.011 \\
Previous surgery & $17(14.2)$ & $2(16.7)$ & 0.810 \\
Bilateral horseshoe arm & $74(61.7)$ & $8(66.7)$ & 0.730 \\
Tract extension & & & \\
$\quad$ Supralevator extension & $11(9.2)$ & $3(25.0)$ & 0.089 \\
$\quad$ High blind intersphincteric extension & $9(7.5)$ & $4(33.3)$ & 0.004 \\
Multiple procedures done in single & $59(49.2)$ & $5(41.7)$ & 0.620 \\
$\quad$ admission & & & \\
Residing $>50$ km from the hospital & $21(17.5)$ & $8(66.7)$ & $<0.0001$ \\
\hline
\end{tabular}

Values are presented as mean \pm standard deviation, number only, or number (\%).

ration of symptoms (more than 1 year), fistulas with high blind intersphincteric extensions, and residing more than $50 \mathrm{~km}$ away from the hospital extending the intervals of postoperative followup at the clinic.

\section{DISCUSSION}

Complete deroofing of fistula tracts has been the textbook surgical procedure until Hanley's conservative procedure was adopted as the standard technique $[3,5,9]$. A modified version $[4,6]$ is now widely practiced, but the basic conception in these procedures is that the horseshoe fistulous extensions from the primary sepsis will resolve spontaneously once the primary pathology is removed. Reasons for recurrence in horseshoe fistula are presumed to be failure to identify the primary lesion and insufficient drainage. We believe another reason is that the lateral extensions from the primary lesion is oftentimes underestimated. Recurrence has been documented in cases where the lateral extensions later required deroofing which was not performed at the initial surgery [2]. Prior to adopting the 2-staged deroofing fistulotomy method as our primary choice of treatment, we had experienced recurring fistula with the original Hanley's procedure in which the lateral extensions were not deroofed, consequently transitioning to our present surgical method. In the present study, we have also experienced several recurrences caused by inadequate drainage of the extended arms, while the primary lesion itself has healed, suggesting the removal and drainage of the secondary pathology is just as crucial as dealing with the primary pathology.

In our study, patients with a symptom duration of more than 1 year were significantly at risk for recurrence. Fistulas with a long history of recurrent infection may possess lateral extensions in which tissue is partially scarred. We speculate this fibrotic area 
lacks the proper blood flow to heal, thus leaving a pocket of unhealed fistula that may subsequently recur. The recurrences in these long-duration fistulas in our study were only on the surface and resolved with an additional procedure to remove the pathology. The single patient who had been identified with a recurrent primary lesion had a supralevator and high blind intersphincteric extension at the initial surgery. Recurrence emerged more than 3 years after the final surgery, and the recurrent sepsis occurred as a deep-lying abscess with an upward intersphincteric extension, suggesting the recurrence was caused by a residual upward extension.

In the patients who live far from the hospital and whose interval between postoperative visits to the clinic was 4 weeks, premature closure of the wound was suspected as the cause for recurrence. However, the recurrence rate in those who live far was $25.8 \%$, indicating not all patients with long intervals between clinic visits recur. As we felt these results do not justify shortening the intervals for patients who take long hours to visit the clinic, sometimes using air transportation, extra care was taken to educate these patients to treat their wounds properly before discharge.

Incontinence can easily occur when fistulotomy of the intersphincteric tract causes loss of internal sphincter beyond the dentate line. We felt this tended to happen in the original Hanley's procedure, which drains the deep-seated primary lesion and divides the sphincter muscle in a single procedure. However, when division of the sphincter muscle is delayed and performed after the surrounding tissue has healed and become solid, we feel fistulotomy can be done properly and the severed ends of the sphincter muscles do not separate widely, leaving continence intact. Delayed fistulotomy using a cutting seton as in the modified Hanley's procedure has been reported to be well tolerated in terms of incontinence $[7,8,10,11]$.

The minimum interval of 5 days between the first and second definitive surgery was determined based on experience seeing that the wound was stable after 5 days. A previous report performing delayed posterior midline fistulotomy on horseshoe fistula using a cutting seton pulling through the muscles at 5 to 8 days yielded functional results within acceptable range [2], showing this time frame to be adequate.

The primary lesion is accessible by dissecting deeper behind the sphincter complex severing the anococcygeal ligament or its superficial external sphincter extension. Hanley et al. [1] has advised not to sever the superficial external sphincter between its coccygeal origin and the anus, to avoid anterior displacement of the anal canal, but we have observed no deformity in the anus in any of our patients. In many patients, when identifying the anococcygeal ligament and its external sphincter extension, the coccygeal end of the anococcygeal ligament appears as a thin bundle of fiber, not providing much support for the anal canal. We have found this to be the case, especially in elderly patients. Reports on dissection of cadavers have revealed the anococcygeal ligament to consist of 2 different layers, in which the superficial layer is a thin bundle extending between the coccyx and external sphincter [12, 13]. One of the reports also stated the superficial layer is unlikely to provide a stable mechanical support to maintain a suitable positioning of the anorectum to the coccyx [12]. The literature along with our findings suggest that the ligament does not provide adequate support for the anal canal, indicating that the severance of this structure does not directly lead to incontinence.

Crossing the anococcygeal ligament makes it easier to locate the sphincter complex covering the primary lesion under the extended view. A wider field of vision makes it easier to confirm the muscle structures associated with continence. The origin of the infection has long been considered to be located in the posterior extrasphincteric space [3, 14], but a new concept has emerged illustrating an intersphincteric space to be the origin $[15,16]$. Advances in radiologic imaging such as magnetic resonance imaging may have helped facilitate the introduction of this new concept. We also speculate the reason why this concept had not been recognized for a long time may be because the posterior midline incision, which only permits a limited view of the muscle structures surrounding the origin, may easily lead to misjudgment of the sphincter layer. Without identifying the deep external sphincter, drainage of the deep space may be incomplete. In our procedure, the entire fistula tract along with the primary lesion can be identified and treated rather easily when the anorectal anatomy can be viewed directly with a wide incision.

With smaller incisions and shorter hospital stays becoming more common, a large incision with multiple operations may seem like a backward concept, but the need for an easier way to achieve better results has long been searched. We speculate that our surgical procedure may produce good results especially in cases of horseshoe fistula with long or complicated extended arms. There have been previous literature describing fistulotomy of the horseshoe fistula extensions along with drainage of the deep sepsis reporting to be successful with few recurrences [2, 17], but not on a large scale as in this report. One of the reports performed fistulotomy of the lateral tracts with seton division of the sphincter muscle, but $20 \%$ of the patients required reoperation [2].

There are several limitations to this study. It is a retrospective series from a single institution, with no comparison with other surgical procedures. Data recording did not include imaging with magnetic resonance scan. The lack of objective data such as postoperative anal manometry is also a limitation. However, it is difficult to justify this examination when patients have no symptoms relevant to incontinence. Furthermore, although a single-center analysis, data extracted were of operations conducted by multiple surgeons. Despite these limitations, this study describes good outcomes of a surgical method easily reproduced. In addition, the strengths of this study include the large sample size of a single surgical method performed on a fairly rare pathological condition, which most likely would not be possible at any other institution. 
In conclusion, by dividing the procedure into 2 parts, complete deroofing fistulotomy can be performed without incontinence. This procedure for horseshoe fistula provides a wider exposure to the origin of infection than the conventional approach and a proper draining route, thus avoids recurrence.

\section{CONFLICT OF INTEREST}

No potential conflict of interest relevant to this article was reported.

\section{REFERENCES}

1. Hanley PH, Ray JE, Pennington EE, Grablowsky OM. Fistula-inano: a ten-year follow-up study of horseshoe-abscess fistula-inano. Dis Colon Rectum 1976;19:507-15.

2. Pezim ME. Successful treatment of horseshoe fistula requires deroofing of deep postanal space. Am J Surg 1994;167:513-5.

3. Hanley PH. Conservative surgical correction of horseshoe abscess and fistula. Dis Colon Rectum 1965;8:364-8.

4. Browder LK, Sweet S, Kaiser AM. Modified Hanley procedure for management of complex horseshoe fistulae. Tech Coloproctol 2009;13:301-6.

5. Goligher JC, Duthie HL, Nixon HH. Surgery of the anus, rectum and colon. 3rd ed. London: Baillière Tindall; 1975.

6. Leventoğlu S, Ege B, Menteş BB, Yörübulut M, Soydan S, Aytaç B. Treatment for horseshoe fistula with the modified Hanley procedure using a hybrid seton: results of 21 cases. Tech Coloproctol 2013;17:411-7.

7. Garcia-Aguilar J, Belmonte C, Wong WD, Goldberg SM, Madoff RD. Anal fistula surgery. Factors associated with recurrence and incontinence. Dis Colon Rectum 1996;39:723-9.

8. Held D, Khubchandani I, Sheets J, Stasik J, Rosen L, Riether R. Management of anorectal horseshoe abscess and fistula. Dis Colon Rectum 1986;29:793-7.

9. Keighley MR, Williams NS, Sagar P. Keighley \& Williams' surgery of the anus, rectum and colon. 4th ed. Boca Raton (FL): CRC Press; 2019.

10. Ustynoski K, Rosen L, Stasik J, Riether R, Sheets J, Khubchandani IT. Horseshoe abscess fistula. Seton treatment. Dis Colon Rectum 1990;33:602-5.

11. Hanley PH. Reflections on anorectal abscess fistula: 1984. Dis Colon Rectum 1985;28:528-33.

12. Jin ZW, Hata F, Jin Y, Murakami G, Kinugasa Y, Abe S. The anococcygeal ligaments: cadaveric study with application to our understanding of incontinence in the elderly. Clin Anat 2015;28: 1039-47.

13. Kinugasa Y, Arakawa T, Abe S, Ohtsuka A, Suzuki D, Murakami G, et al. Anatomical reevaluation of the anococcygeal ligament and its surgical relevance. Dis Colon Rectum 2011;54:232-7.

14. Courtney $H$. The posterior subsphincteric space; its relation to posterior horseshoe fistula. Surg Gynecol Obstet 1949;89:222-6.

15. Kurihara H, Kanai T, Ishikawa T, Ozawa K, Kanatake Y, Kanai S, et al. A new concept for the surgical anatomy of posterior deep complex fistulas: the posterior deep space and the septum of the ischiorectal fossa. Dis Colon Rectum 2006;49(10 Suppl):S37-44.

16. Zhang H, Zhou ZY, Hu B, Liu DC, Peng H, Xie SK, et al. Clinical significance of 2 deep posterior perianal spaces to complex cryptoglandular fistulas. Dis Colon Rectum 2016;59:766-74.

17. Inceoglu R, Gencosmanoglu R. Fistulotomy and drainage of deep postanal space abscess in the treatment of posterior horseshoe fistula. BMC Surg 2003;3:10. 